\title{
Demographic disparities in delay of definitive chemoradiation for anal squamous cell carcinoma: a nationwide analysis
}

\author{
Stephen J. Ramey ${ }^{1,2}$, Benjamin J. Rich ${ }^{2}$, Deukwoo Kwon ${ }^{3}$, Eric A. Mellon ${ }^{2}$, Aaron Wolfson ${ }^{2}$, Lorraine \\ Portelance $^{2}$, Raphael Yechieli ${ }^{2}$ \\ ${ }^{1}$ Department of Radiation Oncology, Georgia Cancer Center, Augusta University, Augusta, Georgia, USA; ${ }^{2}$ Department of Radiation Oncology, \\ ${ }^{3}$ Biostatistics and Bioinformatics Core, Sylvester Comprehensive Cancer Center/University of Miami Miller School of Medicine, Miami, USA \\ Contributions: (I) Conception and design: SJ Ramey, EA Mellon, A Wolfson, L Portelance, R Yechieli; (II) Administrative support: SJ Ramey, R \\ Yechieli; (III) Provision of study materials or patients: SJ Ramey, R Yechieli; (IV) Collection and assembly of data: SJ Ramey, BJ Rich, D Kwon, R \\ Yechieli; (V) Data analysis and interpretation: All authors; (VI) Manuscript writing: All authors; (VII) Final approval of manuscript: All authors. \\ Correspondence to: Raphael Yechieli, MD. 1475 NW 12th Avenue, Suite 1500, Miami, FL 33136, USA. Email: ryechieli@med.miami.edu.
}

Background: Prolonged time to treatment initiation (TTI) for patients with curable anal cancer may reduce tumor control. This study investigated demographic disparities in TTI for patients receiving definitive chemoradiation (CRT) for anal squamous cell carcinoma (A-SCC).

Methods: Adult patients with A-SCC diagnosed from 2004 to 2014 and treated with definitive CRT were identified in the National Cancer Database (NCDB). TTI was defined as days from diagnosis to start of CRT. A negative binomial regression model estimated predicted TTI (pTTI) values. Cox proportional hazards model evaluated the impact of TTI on overall survival (OS).

Results: Overall, 12,546 patients were included with 9\% Non-Hispanic Black patients and 4\% Hispanic patients. Multivariable analysis (MVA) showed that pTTI varied significantly by race/ethnicity with NonHispanic Black patients having a pTTI of 50 vs. 38 days for Non-Hispanic White patients [relative risk (RR), 1.21; 95\% confidence interval (CI), 1.17-1.25]. For Hispanic patients, pTTI was 48 days, significantly longer than that of Non-Hispanic White patients (RR, 1.19; 95\% CI, 1.14-1.24). Gender, insurance status, education level, urban category, distance to reporting facility, treatment facility type, intensity-modulated radiation therapy (IMRT)/proton use, T/N classification, and comorbidity status were all also associated with significant variation in TTI. TTI was not independently associated with changes in OS on MVA [hazard ratio (HR), 0.999; 95\% CI, 0.997-1.002].

Conclusions: Non-Hispanic Black and Hispanic patients have longer delays in starting definitive CRT for A-SCC. While TTI was not associated with OS, future analyses should explore the impact of TTI on local control, metastases, and patient-reported outcomes.

Keywords: Anus neoplasms; chemoradiotherapy; radiotherapy; healthcare disparities; treatment delay

Submitted Jun 08, 2018. Accepted for publication Jul 23, 2018.

doi: 10.21037/jgo.2018.08.07

View this article at: http://dx.doi.org/10.21037/jgo.2018.08.07

\section{Introduction}

In 2016, there were an estimated 8,080 new cases of anal cancer in the US and 1,080 deaths (1). The incidence of anal squamous cell carcinoma (A-SCC) has been increasing in the US over the last 30 years (2). The standard therapy for A-SCC is definitive radiation therapy (RT) with concurrent 5-fluorouracil and mitomycin-C (3). This treatment results in excellent outcomes, with one recent cohort study finding a 4-year overall survival (OS) of $86 \%$ in patients with nonmetastatic disease (4).

Black patients with anal cancer have a lower 5-year relative survival (56\%) compared to White patients (67\%) (1). Although relative survival of Hispanics with anal cancer 
has not been reported, analysis from other primary disease sites indicates that Hispanic patients have a lower 5-year survival (5). The etiology of the racial discrepancy in survival of patients with anal cancer is likely multifactorial; for example, Black patients diagnosed with non-metastatic A-SCC are less likely to receive RT than their White patient counterparts (6). Another possible etiology is an increased time to treatment initiation (TTI). Increased TTI, defined as the duration between diagnosis and treatment, is independently associated with worse OS in head and neck cancer (7) and breast cancer (8). However, disparities in initial treatment delay and the impact of treatment delay on oncologic or OS outcomes have not been previously studied in A-SCC.

A-SCC and head and neck cancer share similar etiologies and histology. Human papillomavirus (HPV) infection has been shown to be present in $93 \%$ of A-SCC tumors (9) and $64 \%$ of oropharyngeal cancer tumors (10) although HPV positivity is lower in non-oropharyngeal head and neck cancers (11). In addition, smoking is a strong independent risk factor for both A-SCC (9) and head and neck cancer (12). More than $90 \%$ of head and neck cancers are squamous cell carcinoma; as a result, head and neck cancer and A-SCC have a common histology and similar potential doubling time (13). Since these two cancers share similar pathogenesis, it would be expected that the survival consequences of delayed treatment initiation observed in head and neck cancer might also be seen in A-SCC.

TTI has been shown to be increased in Black patients compared to White patients in breast cancer $(8,14)$, prostate cancer (15), and head and neck cancer (16). Likewise, longer TTI's have been noted in Hispanic patients with head and neck cancer when compared to Non-Hispanic patients (16). However, the socioeconomic and/or demographic factors influencing TTI in patients with A-SCC have never been described. A variety of other factors [e.g., transitions of care (16), use of intensity-modulated radiation therapy (IMRT) (17), treatment at academic centers (16), treatment facility volume (18), socioeconomic status (16), urban or rural status $(15)$, and insurance status $(16,17,19)]$ have also been associated with TTI in previous analyses from other primary disease sites but have not been previously assessed in A-SCC. We hypothesized that TTI of chemoradiation (CRT) for A-SCC is longer for Non-Hispanic Black and Hispanic patients when compared to Non-Hispanic White patients. In the current study, we analyzed A-SCC patients in the National Cancer Database (NCDB) treated with definitive CRT to determine patient characteristics that affect TTI. Additionally, a secondary analysis was performed to determine whether increasing TTI was associated with worse OS.

\section{Methods}

The University of Miami Institutional Review Board (IRB) deemed this study exempt from review since only de-identified data was used. The American College of Surgeons granted access to the 2014 NCDB participant user file. The NCDB is a joint project of the Commission on Cancer $(\mathrm{CoC})$ of the American College of Surgeons and the American Cancer Society. The CoC's NCDB and the hospitals participating in the CoC NCDB are the source of the de-identified data used herein; they have not verified and are not responsible for the statistical validity of the data analysis or the conclusions derived by the authors. Over 1,500 programs submit data to the NCDB, representing approximately $70 \%$ of new cancer cases in the US and Puerto Rico (20). For anal cancer, NCDB reporting rates are estimated to be even higher at $87 \%$ of cases (21). Data collected by NCDB undergo a variety of automated tests, and institutions are required to have regular reviews to improve data accuracy $(22,23)$. Furthermore, at least a $90 \%$ follow-up rate for 5 years is required of reporting institutions (22).

\section{Patient selection}

There were 54,069 adult patients (ages 18 and above) diagnosed with anal cancer between 2004 and 2014 who were analyzed for this study. Figure 1 shows sequential exclusion criteria and the number of patients excluded by each criterion. Patients with American Joint Committee on Cancer (AJCC) clinical group stage 3 without subcategorization (i.e., A versus B), in situ disease without nodal metastases, or distant metastases were excluded. The International Classification of Diseases for Oncology, 3rd Edition (ICD-O-3) site codes were used to define histology (24). Only patients treated with concurrent CRT (defined as chemotherapy and RT starting within 7 days of each other) were included. Thus, patients treated with surgery, chemotherapy alone, RT alone, brachytherapy alone, radiation of unspecified type, or non-concurrent CRT were excluded. Patients receiving non-standard radiation modalities for anal cancer treatment (e.g., orthovoltage, strontium, Gamma Knife, etc.) were excluded. Finally, patients with an unknown TTI, TTI of 0 days, or TTI 


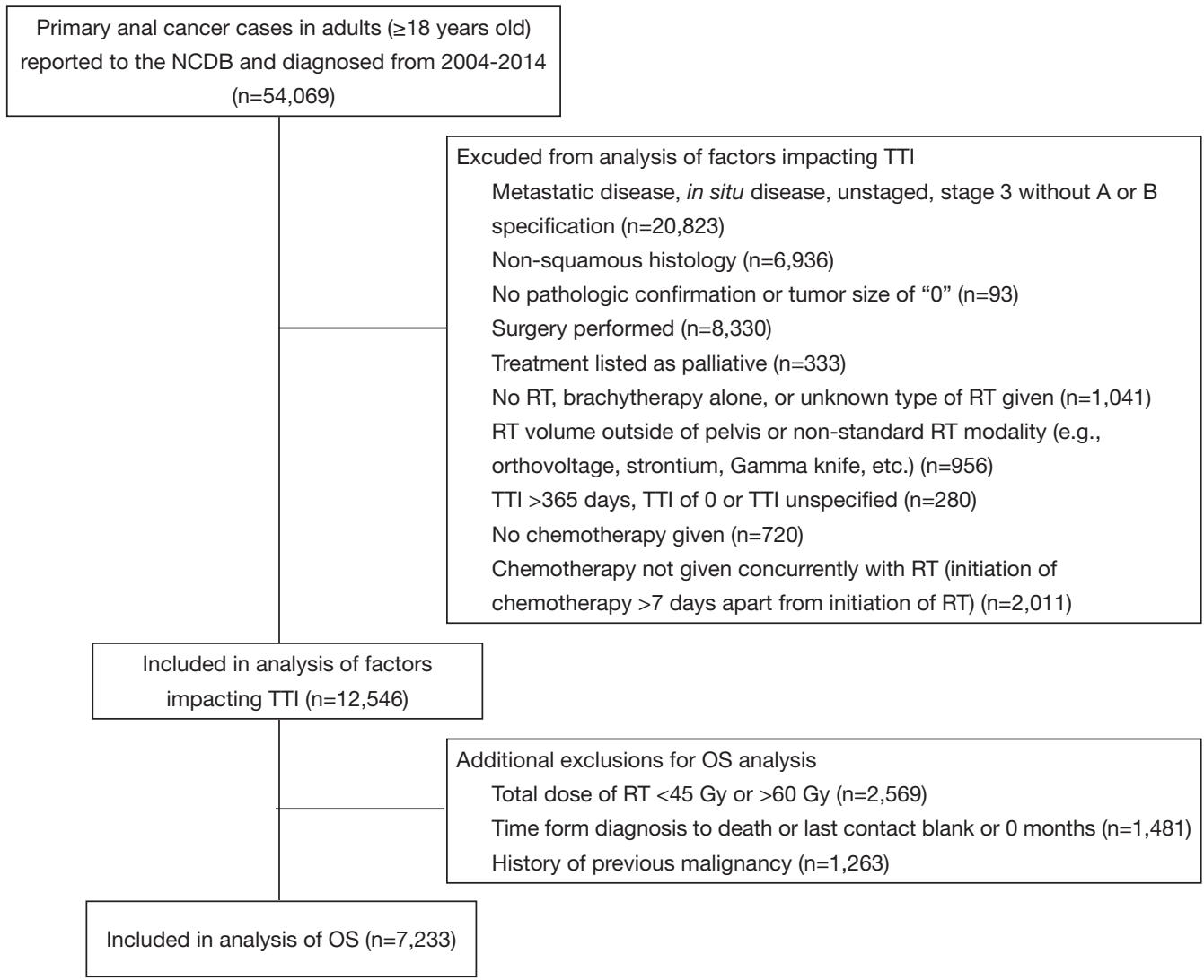

Figure 1 Diagram of exclusion criteria with number of patients excluded by each subsequent criterion. NCDB, National Cancer Database; OS, overall survival; RT, radiation therapy; TTI, time to treatment initiation.

greater than 365 days (given concern that a TTI of this length could represent coding error) were excluded (7).

Additional exclusion factors applied prior to the OS analysis are also displayed in Figure 1. Patients were excluded if time from diagnosis to death was unknown or listed as "0" months. This criterion resulted in exclusion of all patients diagnosed in 2014 because of the NCDB's policy of not reporting survival data for patients with less than 1 year of follow-up. As has been previously recommended for OS analyses using the NCDB, patients with a history of prior malignancy were excluded (25). Patients who received a non-standard RT dose of less than 45 Gy or greater than 60 Gy were also excluded from the OS analysis.

\section{Variable definitions}

Variables used for this study are defined as follows. Percent of high school graduates and median household income quartiles were defined by 2012 American Community
Survey data based on the patient's zip code of residence at diagnosis. Urban-rural categorization similarly relied on United States Department of Agriculture Economic Research Service data from 2013. The distance from the patient's home to the reporting center was defined as the "greatest circle" distance in miles from the center of the patient's zip code at diagnosis to the reporting center. A transition in care was defined as a patient diagnosed at one facility and treated at another. Reporting centers were divided into tertiles based on the number of cases reported by that center between 2004-2014 as previously described (26). In the final cohort for this study, 20 patients were treated with protons and were analyzed with the IMRT cohort. Comorbid conditions as described by the Charlson/Deyo comorbidity index are derived using a weighted score based on the presence and severity of ten diagnosis codes (27). Charlson/Deyo scores are condensed by the NCDB to 0 , 1 , and 2 (with 2 representing all cases with a score $>1$ due to the small proportion of patients with scores $>2)(25)$. 
AJCC clinical stage and TNM classification were reported based on the edition of the AJCC Cancer Staging Manual in place at the time of diagnosis (i.e., 6th edition for patients diagnosed from 2004 to 2009 and 7th edition for patients diagnosed from 2010 to 2014). No changes to AJCC anal cancer staging were made between these editions. TTI was defined as days from clinical or histological diagnosis (defined as the first definitive mention of a cancer diagnosis in the medical record) to start of RT or chemotherapy. OS was defined as the number of months from diagnosis to death. Individuals still alive at last follow-up were censored at that time point.

\section{Statistical methods}

Initially, covariates listed in Table 1 were assessed in a univariable analysis (UVA) for effect on TTI. TTI values within each categorical variable were compared using a Kruskal-Wallis nonparametric test. All covariates were incorporated into the multivariable analysis (MVA) assessing TTI variation based on different demographic, treatment and prognostic factors. To improve simplicity of comparisons of differences in TTI by various independent variables, a predicted TTI (pTTI), measured in days, was created as previously described (16) using a negative binomial regression model. Relative risk (RR) of treatment delay values was also compared between individual subcategories to evaluate whether differences were significant between individual covariates.

A subsequent analysis of OS was then performed after additional exclusion criteria (Figure 1) were applied. The Cox proportional hazard model was used to calculate hazard ratios (HRs) and 95\% confidence intervals (CIs) with adjustment for all factors used in the initial MVA of factors associated with TTI (Table 2) with the addition of TTI and radiation dose as independent variables. The proportionality assumption was tested utilizing Schoenfeld residuals. Tests were two-sided, and findings were considered statistically significant if the $\mathrm{P}$ value was $\leq 0.05$. All statistical analyses were performed with statistical software packages SAS/ STAT $^{\circledR}$ version 9.4 (SAS Institute Inc.) or R (version 3.3.1).

\section{Results}

\section{Baseline characteristics and UVA analysis of factors impacting TTI}

A total of 12,546 patients with non-metastatic A-SCC treated with definitive CRT were included in the initial cohort analyzing factors associated with TTI. The median OS of this cohort was 132.9 months. The cohort demographic and prognostic characteristics are described in Table 1 along with the mean and median TTI associated with each characteristic. Additionally, Table 1 includes p-values of each categorical variable's TTI compared using a Kruskal-Wallis nonparametric test. On UVA, median TTI varied significantly by race and ethnicity with Non-Hispanic White and Asian-American and Pacific Islander patients waiting a median of 33 and 35 days for CRT, respectively, compared to Non-Hispanic Black and Hispanic patients waiting a median of 42 and 40 days, respectively, for initiation of CRT $(\mathrm{P}<0.001)$. Patient's insurance status was also associated $(\mathrm{P}<0.001)$ with TTI; patients with private insurance had a median TTI of 32 days compared to 35, 34 and 40 days for patients without insurance, with Medicare, and with Medicaid, respectively. Figure 2 illustrates the distribution of TTI by race and ethnicity (Figure $2 A$ ) as well as by insurance status (Figure 2B). Furthermore, median TTI varied significantly $(\mathrm{P}<0.001)$ by patient distance to reporting facility, gender, medical comorbidities, AJCC clinical tumor classification, and AJCC clinical nodal classification. Patient age was not associated with median TTI. The year of diagnosis had a significant impact on TTI $(\mathrm{P}<0.001)$ : patients diagnosed with A-SCC in 2004 had a median TTI of 30 days, which increased to 35 days for patients diagnosed in 2014. TTI also varied with the education level and median income of the zip code where the patient resided. Treatment characteristics associated with TTI on UVA $(\mathrm{P}<0.001)$ included reporting facility type (i.e., academic, community), reporting facility anal cancer patient volume, reporting facility regional location, radiation treatment with IMRT or protons, and a transition of care during diagnosis and treatment.

\section{MVA of factors impacting TTI}

For MVA, a pTTI was generated using a negative binomial regression model (Table 2). On MVA, race and ethnicity remained a significant contributor to treatment delays. The pTTI of Non-Hispanic Black patients was 49.5 days and of Hispanic patients was 48.0 days, both significantly longer $(\mathrm{P}<0.001)$ than the 37.8 days' pTTI of Non-Hispanic White patients. Asian-American and Pacific Islander patients had a pTTI of 42.1, but this was not statistically significant relative to Non-Hispanic White patients. Male patients had an increased pTTI of 
Table 1 Time to treatment initiation UVA cohort: patient demographic, treatment/treatment center, and prognostic characteristics with associated mean and median TTI

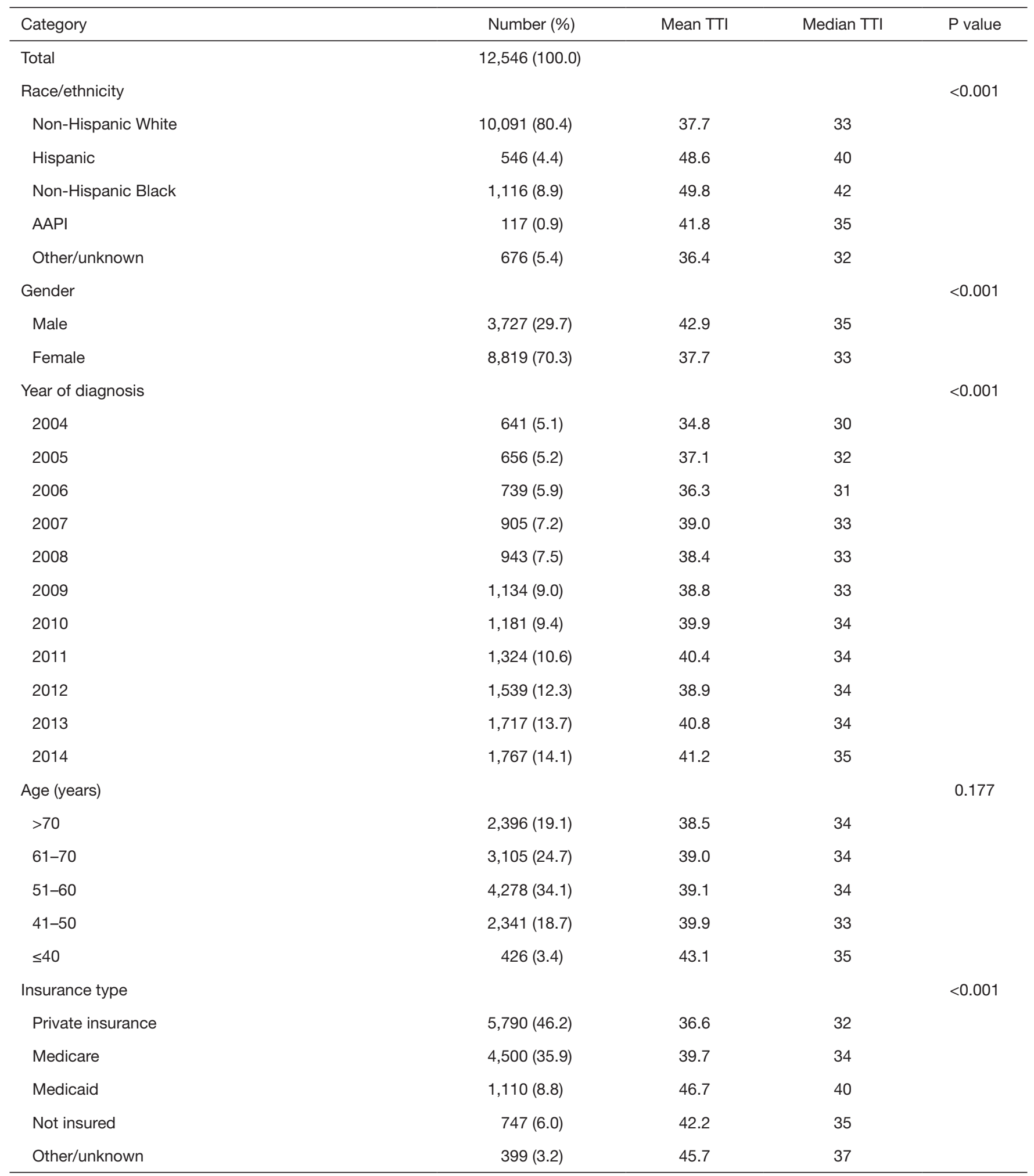

Table 1 (continued) 
Table 1 (continued)

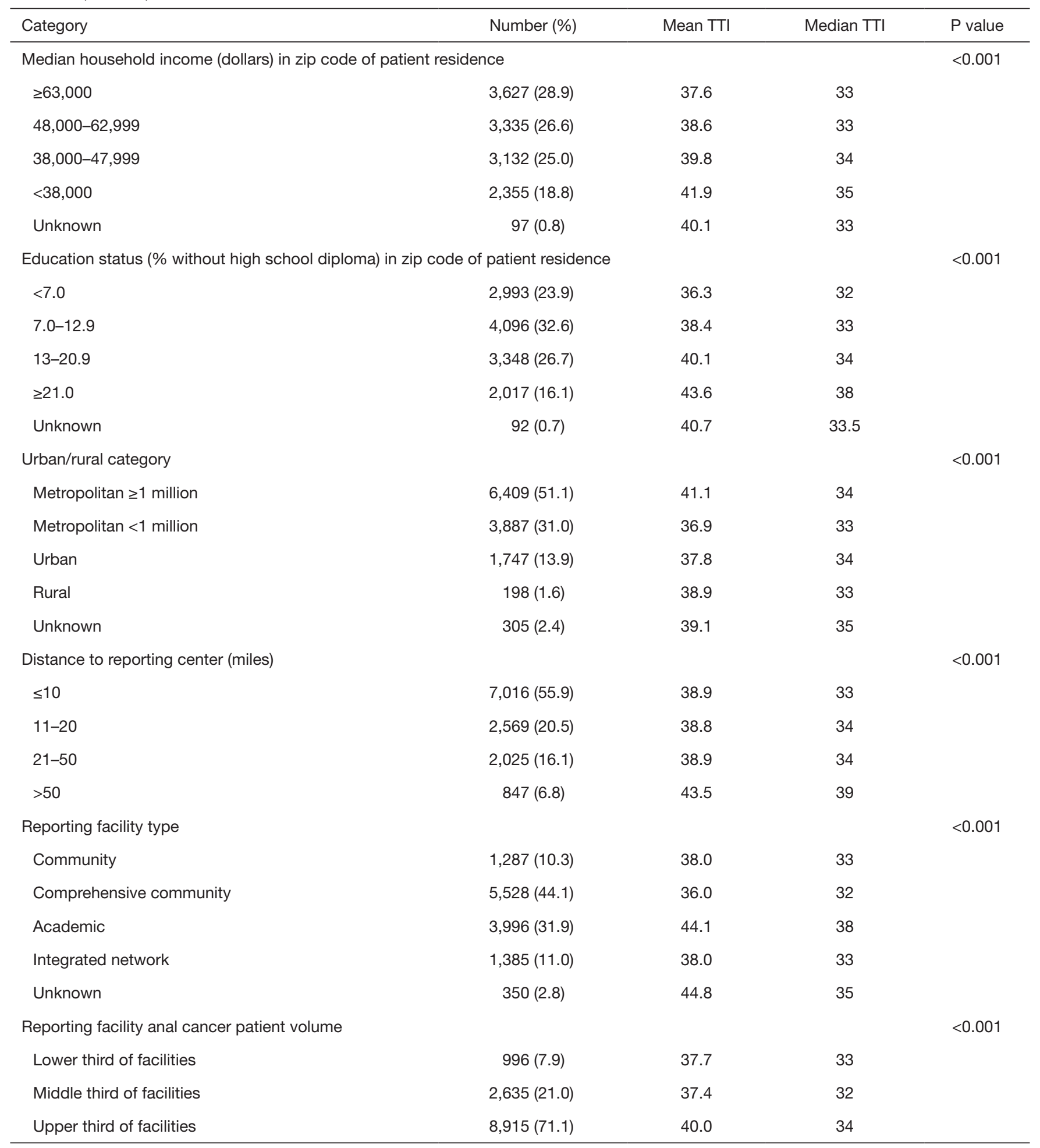

Table 1 (continued) 
Table 1 (continued)

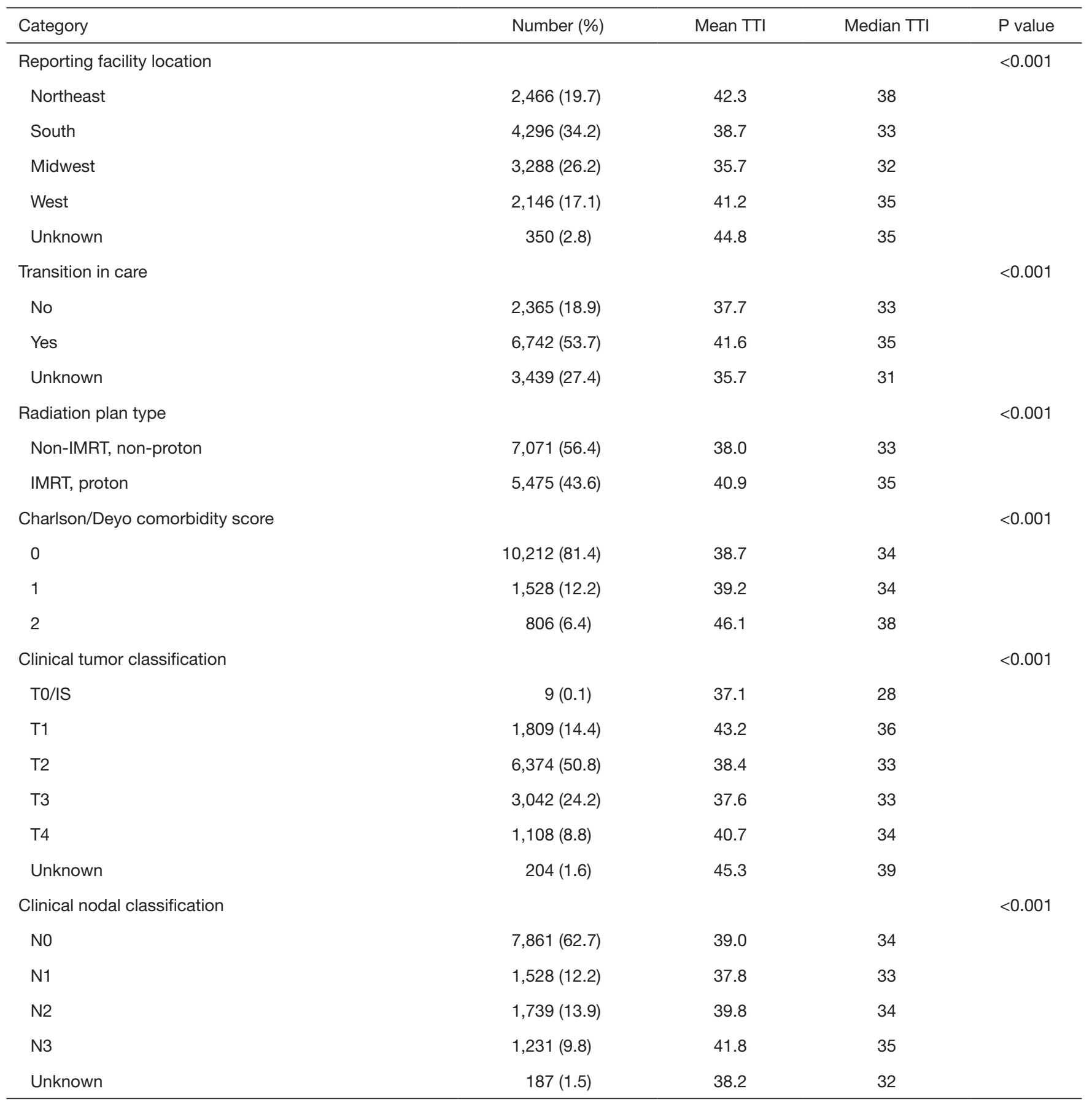

$\mathrm{P}$ values reported from Kruskal-Wallis test. UVA, univariable analysis; TTI, time to treatment initiation; AAPI, Asian-American and Pacific Islander; IMRT, intensity-modulated radiation therapy; IS, in situ. 
Table 2 Multivariable analyses assessing impact of demographic, treatment/treatment center, and prognostic factors on TTI

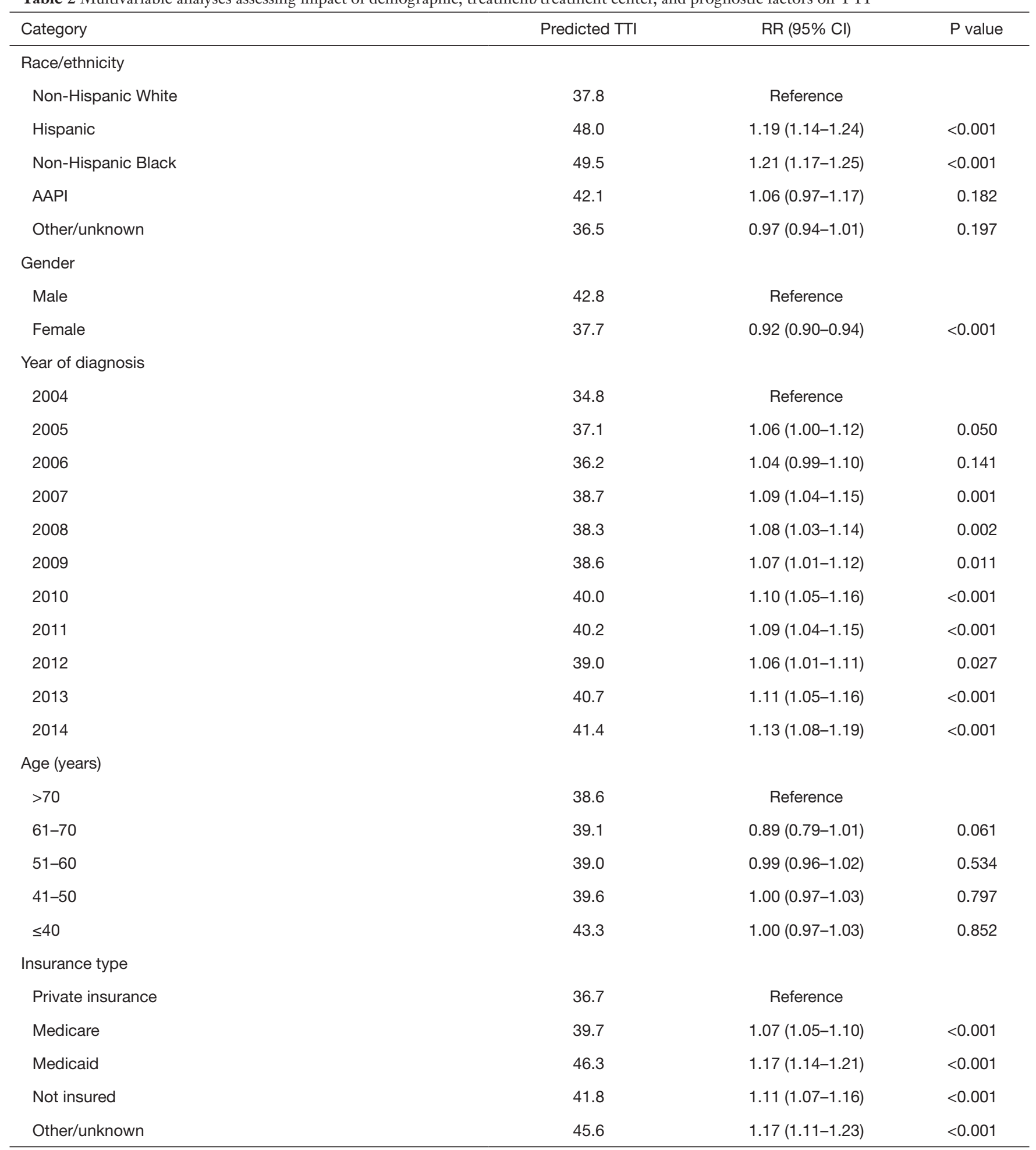

Table 2 (continued) 
Table 2 (continued)

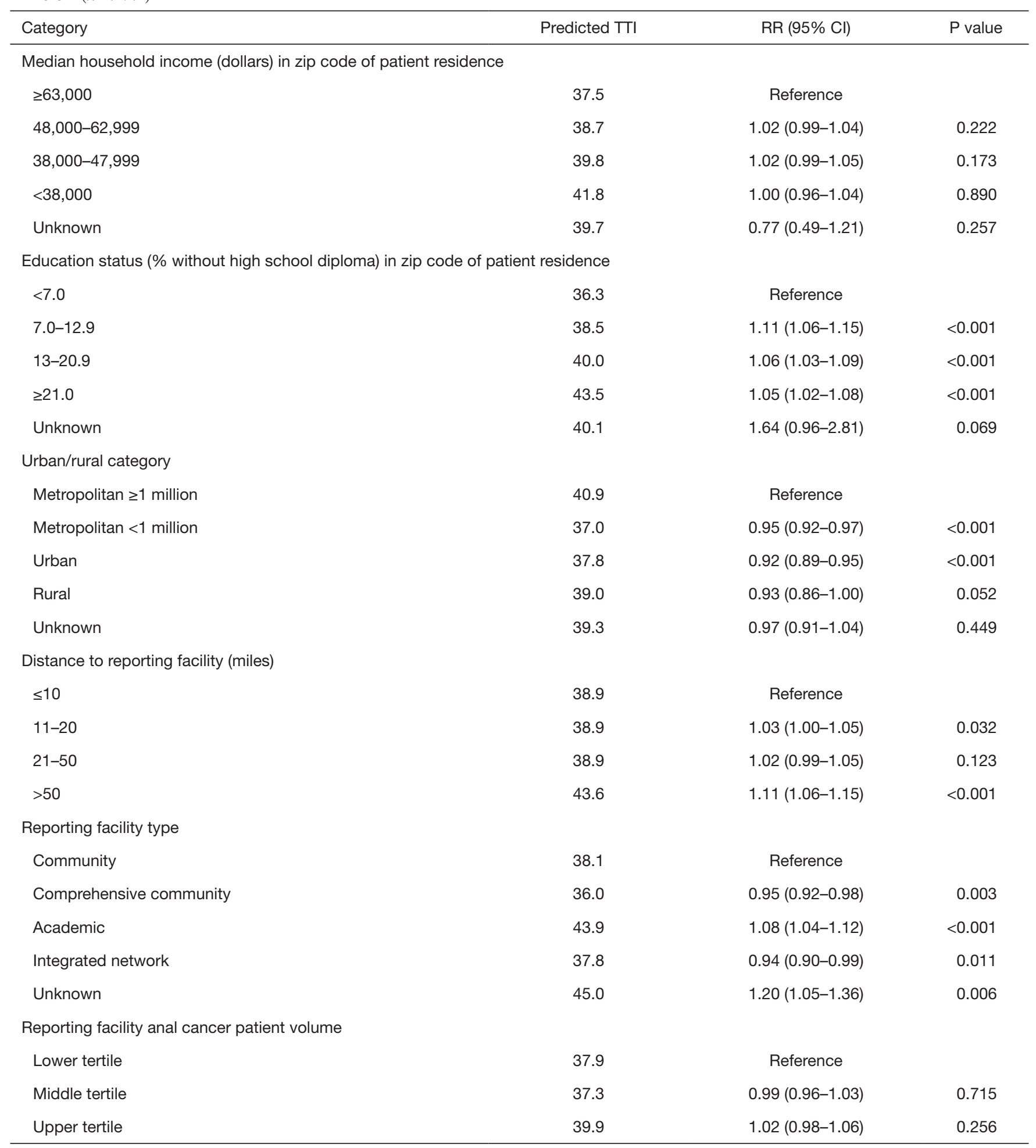

Table 2 (continued) 
Table 2 (continued)

\begin{tabular}{|c|c|c|c|}
\hline Category & Predicted TTI & RR $(95 \%$ Cl) & $P$ value \\
\hline \multicolumn{4}{|l|}{ Transitions in care } \\
\hline No & 37.7 & Reference & \\
\hline Yes & 41.5 & $1.13(1.11-1.16)$ & $<0.001$ \\
\hline Unknown & 35.7 & $1.01(0.98-1.05)$ & 0.402 \\
\hline \multicolumn{4}{|l|}{ Radiation plan type } \\
\hline Non-IMRT, non-proton & 37.8 & Reference & \\
\hline IMRT, proton & 41.0 & $1.06(1.04-1.08)$ & $<0.001$ \\
\hline \multicolumn{4}{|c|}{ Charlson/Deyo comorbidity score } \\
\hline 0 & 38.7 & Reference & \\
\hline 1 & 39.2 & $1.01(0.98-1.04)$ & 0.560 \\
\hline 2 & 45.9 & $1.09(1.05-1.13)$ & $<0.001$ \\
\hline \multicolumn{4}{|l|}{ Clinical tumor classification } \\
\hline $\mathrm{T} 1$ & 43.1 & Reference & \\
\hline T2 & 38.5 & $0.88(0.86-0.90)$ & $<0.001$ \\
\hline T3 & 37.6 & $0.84(0.82-0.87)$ & $<0.001$ \\
\hline T4 & 40.4 & $0.91(0.87-0.94)$ & $<0.001$ \\
\hline TO/IS & 37.9 & $0.85(0.61-1.18)$ & 0.323 \\
\hline Unknown & 44.5 & $0.99(0.91-1.06)$ & 0.695 \\
\hline \multicolumn{4}{|l|}{ Clinical nodal classification } \\
\hline No & 39.0 & Reference & \\
\hline N1 & 37.8 & $0.97(0.94-1.00)$ & 0.032 \\
\hline N2 & 39.8 & $1.00(0.98-1.03)$ & 0.720 \\
\hline N3 & 41.6 & $1.01(0.98-1.04)$ & 0.494 \\
\hline Unknown & 38.5 & $0.99(0.92-1.07)$ & 0.877 \\
\hline
\end{tabular}

$\mathrm{P}$ values reported from Wald test of zero-inflated Negative Binomial regression model. TTI, time to treatment initiation; RR, relative risk; Cl, confidence interval; AAPI, Asian-American and Pacific Islander; IMRT, intensity-modulated radiation therapy; IS, in situ.

42.8 days compared to 37.7 days for female patients with A-SCC $(\mathrm{P}<0.001)$. Patients with private insurance had a significantly lower pTTI (36.7 days, $\mathrm{P}<0.001)$ relative to patients with Medicare (39.7 days), with Medicaid (46.3 days), or without insurance (41.8 days). More advanced AJCC clinical tumor classification (i.e., T2, T3, T4) was associated with a decreased pTTI. Although more advanced tumor classification was independently associated with reduced treatment delay, only AJCC clinical nodal classification of N1 was associated with a significant decrease in pTTI compared to patients with N0 disease.
Additional patient characteristics associated with prolonged pTTI included a high Charlson/Deyo comorbidity score and residing in a zip code with a low proportion of high school graduates. Patients with a Charlson/Deyo comorbidity score of 2 had a pTTI of 45.9 compared to 38.7 for patients with a Charlson/Deyo comorbidity score of $0(\mathrm{P}<0.001)$. In contrast to the UVA, median income of the zip code of the patient's residence in MVA was not associated with pTTI. Treatment facility characteristics associated with an elevated pTTI included academic reporting facilities, which had a pTTI of 43.9 days 


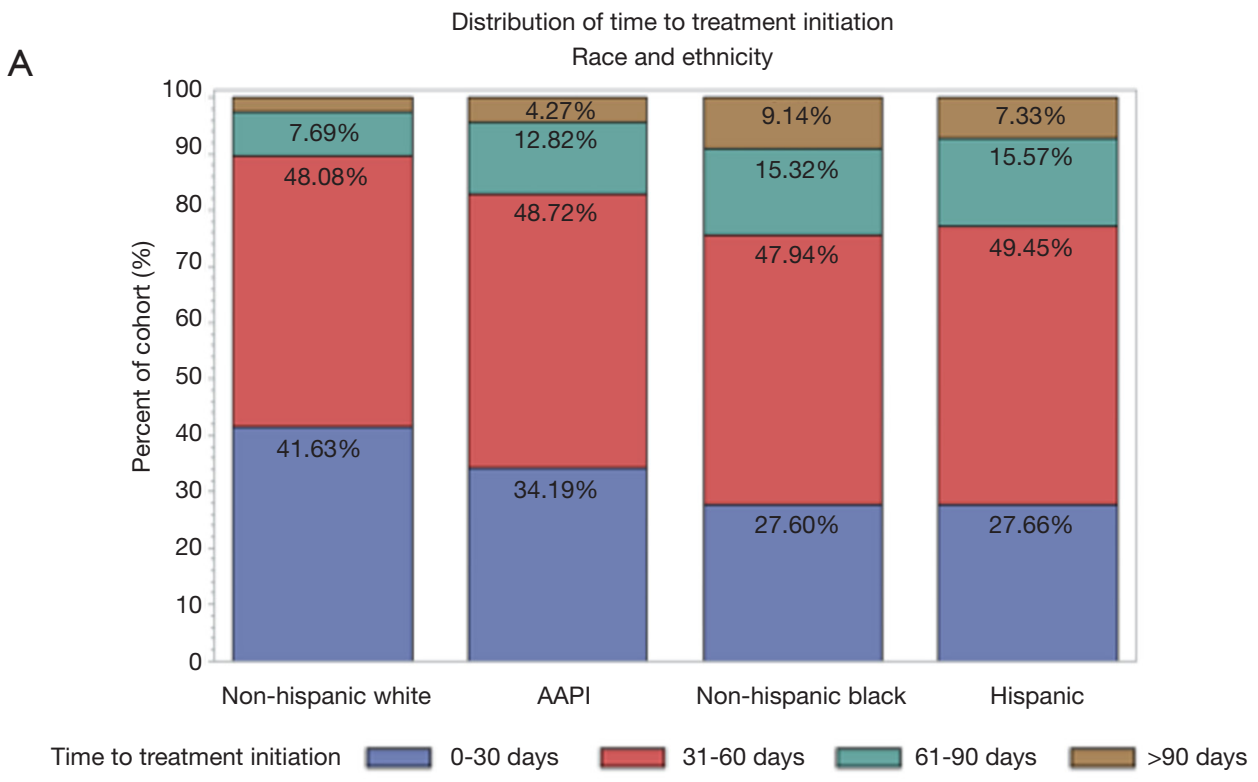

B

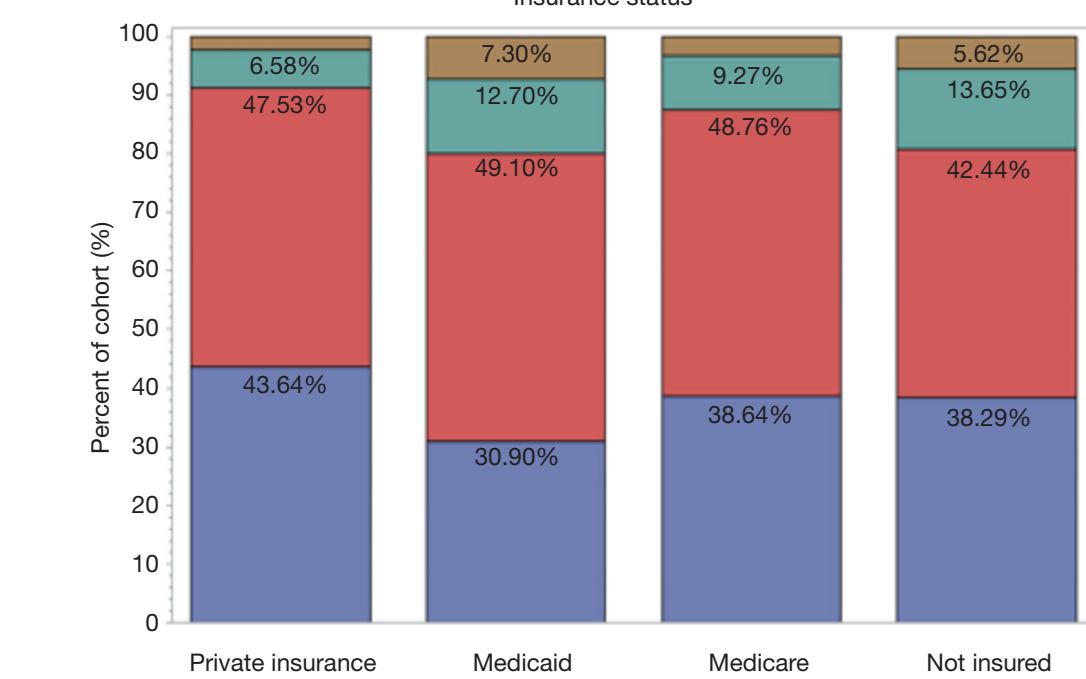

Time to treatment initiation

0-30 days

$31-60$ days

$61-90$ days

$>90$ days

Figure 2 Distribution of time to treatment initiation by race and ethnicity (A) and insurance status (B). AAPI, Asian-American and Pacific Islander.

compared to 38.1 days for community reporting facilities $(\mathrm{P}<0.001)$. Treatment at a reporting facility greater than 50 miles away from the patient's zip code of residence was associated with an elevated pTTI $(\mathrm{P}<0.001)$. Although reporting facility anal cancer patient volume was significant on UVA, this factor was not significant on MVA. Similar to the UVA, treatment involving a transition of care or RT modalities of IMRT or proton therapy was associated with a significant increase in pTTI $(\mathrm{P}<0.001)$.

The year of diagnosis had a significant, independent impact on treatment delay. Patients diagnosed in 2004 had a pTTI of 34.8 days, which increased to 41.4 days in 2014 $(\mathrm{P}<0.001)$. Figure $S 1$ demonstrates the variation in TTI by year within race/ethnicity (Figure $S 1 A$ ), insurance statuses (Figure S1B), reporting facility type (Figure S1C), and AJCC clinical tumor classifications (Figure $S 1 D$ ). 


\section{Analysis of TTI and other factors on OS}

A total of 7,233 patients with non-metastatic A-SCC treated with definitive concurrent CRT were included in a secondary cohort for the analysis of factors associated with OS. This cohort included all previously selected patients who had available survival data, were treated with 45 to 60 Gy of RT, and had no history of prior malignancy. Patient demographic, treatment, and reporting facility characteristics are summarized in Table $S 1$ with mean and median TTI. The median follow-up was 50.7 months.

To determine factors independently associated with OS, a Cox proportional hazard model was used to generate adjusted HRs (Table 3). TTI, treated as a continuous variable, was not independently associated with OS with an HR of 0.999 (95\% CI, 0.997-1.002; $\mathrm{P}=0.582$ ). There was no independent impact of race/ethnicity on OS. Patients with Medicare (HR, 1.52; 95\% CI, 1.32-1.75), Medicaid (HR, 1.76; 95\% CI, 1.48-2.09), and without insurance (HR, 1.40; 95\% CI, 1.14-1.71) had inferior OS compared to patients with private insurance. A-SCC patients treated at academic facilities (HR, 0.72; 95\% CI, 0.59-0.88) or comprehensive community cancer programs (HR, 0.83; 95\% CI, 0.69-0.98) and treated with IMRT or proton therapy (HR, 0.82; $95 \%$ CI, 0.73-0.92) had better OS.

\section{Discussion}

This nationwide database analysis of patients with A-SCC treated with concurrent CRT found that Non-Hispanic Black and Hispanic patients have a significantly prolonged TTI when compared to Non-Hispanic White patients. The pTTI for Non-Hispanic Black patients with A-SCC was $31 \%$ longer or, more specifically, 49.5 days in NonHispanic Black patients compared to 37.8 days in NonHispanic White patients. Likewise, the pTTI of 48.0 days for Hispanic patients was significantly longer than that of Non-Hispanic White patients. In our analysis, this difference did not translate into a difference in OS; however, this healthcare disparity deserves further study given the potential impact such delays could have on other endpoints such as A-SCC specific survival, local control, and patientreported outcomes, including psychological distress.

Murphy et al. demonstrated that Black patients with head and neck cancer had a prolonged pTTI compared to White patients, with a pTTI of 30.4 and 33.5 days in White patients and Black patients, respectively (16). In absolute and relative terms, the racial disparity in A-SCC
TTI evaluated in the present study was greater than that previously reported for head and neck cancer. However, the present study included only patients treated with CRT; CRT is associated with a longer TTI compared to surgery in head and neck cancer $(16,28)$. Nonetheless, surgical treatment delays also exist among Black patients with breast, colon, lung, pancreas, and rectal cancers (29). In analyses of data from the SEER-Medicare and NCDB, time to surgery from diagnosis of breast cancer was longer among Black patients in unadjusted analyses of both databases. Hispanic ethnicity was also more common in patients with longer delay intervals in the SEER-Medicare database (8).

Additional patient characteristics independently associated with an increased pTTI in the present study were male gender, lack of private health insurance (i.e., Medicare, Medicaid, uninsured), residing in an area with a low education level, and a Charlson/Deyo comorbidity score of 2. One institution reported that their head and neck cancer patients on Medicaid had worse outcomes and a longer TTI (19). Characteristics associated with lower socioeconomic status (being uninsured, having low education level, and having low income) are commonly cited barriers to healthcare access (30). However, median income was not independently associated with pTTI in the present study.

The treatment factors associated with an increased pTTI following diagnosis with A-SCC in the present study included a transition of care, home address greater than 50 miles from the reporting facility, treatment at academic centers, and use of IMRT or proton therapy. Cancer treatment involving transition of care and/or IMRT has previously been shown to be associated with head and neck cancer TTI delay $(16,17)$. The aggregate pTTI for patients with A-SCC was found to be trending up from 2004 to 2014 , a trend observed in other cancer sites $(16,29,31)$. It is possible that increasing transitions to high-volume centers and utilization of IMRT as recommended by national guidelines (3) may be partly responsible for the increasing pTTI in A-SCC patients observed over the last decade.

Black patients with anal cancer have previously been reported to have a lower relative 5-year survival than White patients (1). Higher mortality rates in Black patients is not unique to anal cancer; Black patients suffer higher mortality rates than White patients from heart disease, cerebrovascular disease, and HIV/AIDS due to potential differences in disease surveillance, treatment, access to care, and biology (32). Males also have lower overall and diseasespecific survival rates from anal cancer relative to females $(1,33,34)$. In this analysis, a link between pTTI and OS 
Table 3 Multivariable analyses assessing impact of demographic, treatment/treatment center, and prognostic factors on overall survival

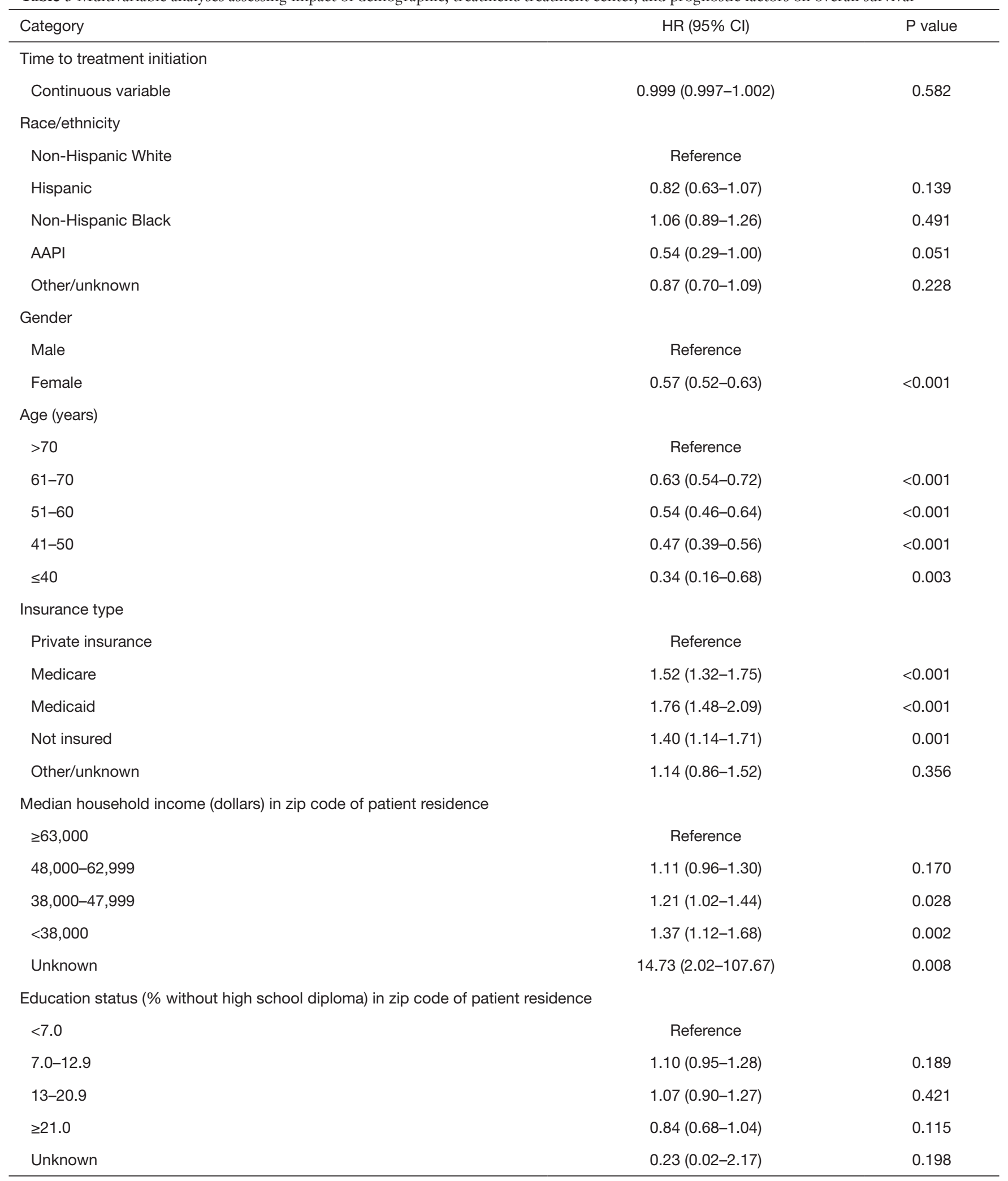

Table 3 (continued) 
Table 3 (continued)

\begin{tabular}{|c|c|c|}
\hline Category & $\mathrm{HR}(95 \% \mathrm{Cl})$ & $P$ value \\
\hline \multicolumn{3}{|l|}{ Urban/rural category } \\
\hline Metropolitan $\geq 1$ million & Reference & \\
\hline Metropolitan $<1$ million & $0.95(0.84-1.07)$ & 0.380 \\
\hline Urban & $0.97(0.81-1.16)$ & 0.734 \\
\hline Unknown & $0.86(0.58-1.28)$ & 0.461 \\
\hline \multicolumn{3}{|c|}{ Distance to reporting center (miles) } \\
\hline$\leq 10$ & Reference & \\
\hline $11-20$ & $0.95(0.83-1.08)$ & 0.443 \\
\hline \multicolumn{3}{|l|}{ Reporting facility type } \\
\hline Community & Reference & \\
\hline Comprehensive community & $0.83(0.69-0.98)$ & 0.032 \\
\hline Academic & $0.72(0.59-0.88)$ & 0.002 \\
\hline Integrated network & $0.89(0.70-1.12)$ & 0.313 \\
\hline Unknown & $1.17(0.55-2.49)$ & 0.690 \\
\hline \multicolumn{3}{|c|}{ Reporting facility anal cancer patient volume } \\
\hline Lower tertile & Reference & \\
\hline Unknown & $1.00(0.83-1.20)$ & 0.979 \\
\hline \multicolumn{3}{|l|}{ Radiation plan type } \\
\hline Non-IMRT, non-proton & Reference & \\
\hline IMRT, proton & $0.82(0.73-0.92)$ & $<0.001$ \\
\hline \multicolumn{3}{|c|}{ Charlson/Deyo comorbidity score } \\
\hline 0 & Reference & \\
\hline 1 & $1.36(1.18-1.56)$ & $<0.001$ \\
\hline 2 & $2.03(1.72-2.41)$ & $<0.001$ \\
\hline \multicolumn{3}{|l|}{ Clinical tumor classification } \\
\hline $\mathrm{T} 1$ & Reference & \\
\hline T2 & $1.34(1.13-1.59)$ & 0.001 \\
\hline
\end{tabular}

Table 3 (continued) 
Table 3 (continued)

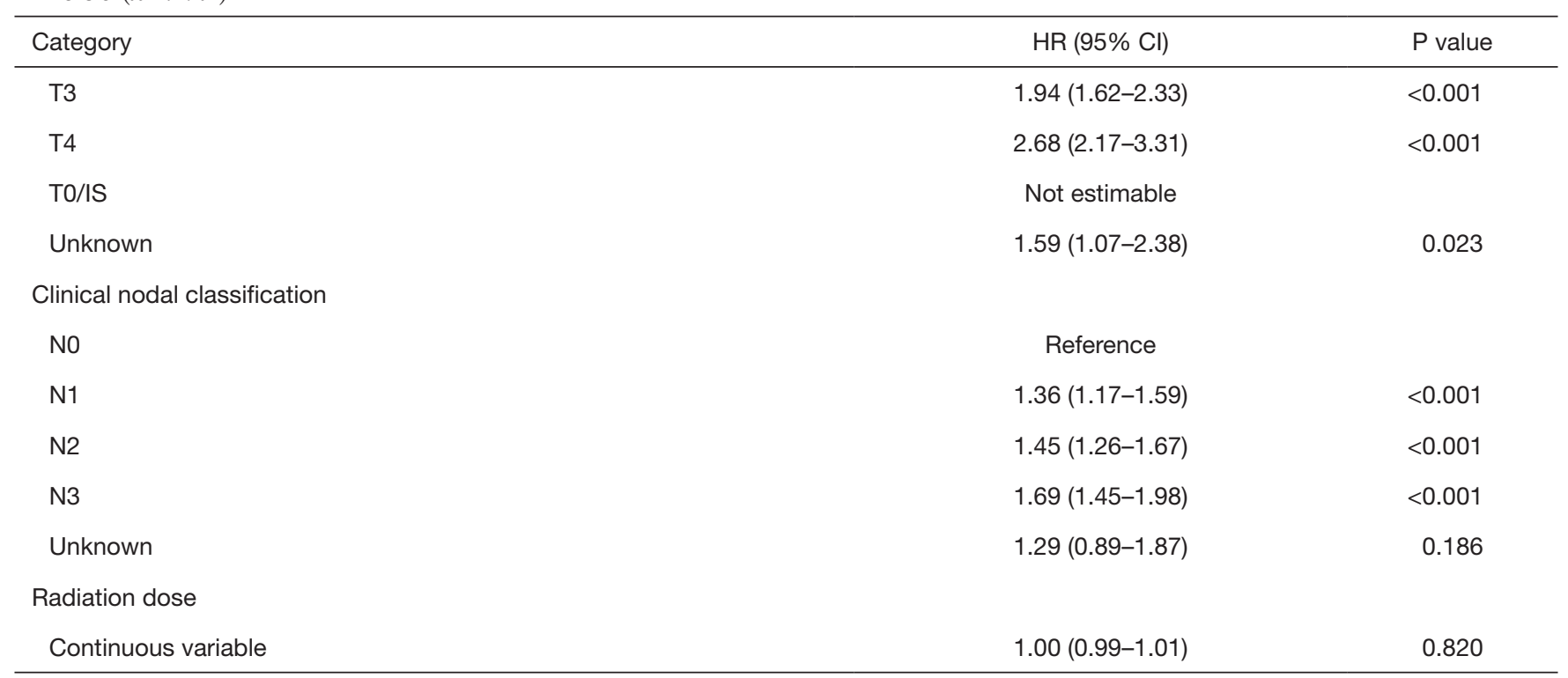

$P$ values from Wald test of Cox proportional hazards regression model. HR, hazard ratio; $\mathrm{Cl}$, confidence interval; AAPI, Asian-American and Pacific Islander; IMRT, intensity-modulated radiation therapy; IS, in situ.

from A-SCC was not elucidated.

Prolonged TTI has been shown in prior studies to be a predictor of worse OS in patients with bladder cancer (18), breast cancer (35), cervical cancer (36), endometrial cancer (31), and head and neck cancer $(7,28)$. Unlike the current study which demonstrated no difference in OS with prolonged TTI, these prior studies all included patients treated with surgery. The data on treatment delay and OS in patients treated exclusively with RT or CRT is scarcer. A retrospective study of patients with cervical cancer treated with curative RT found higher disease-related mortality rates in patients with a longer TTI (37), and another retrospective study of patients with nasopharyngeal carcinoma treated exclusively with RT found worse OS in patients with wait times for treatment over 4 weeks only when the length of radiotherapy also exceeded 10 weeks (38).

Although no relationship between OS and TTI following diagnosis of A-SCC was observed in the present study, there may exist differences in toxicities, patient-reported outcomes, disease-specific mortality, local control, and/ or disease-free survival which could not be analyzed given the lack of information on these endpoints in the NCDB. Delay in RT can result in a deterioration in local control rates, and tumor size can increase significantly even between simulation and treatment $(39,40)$. In a pooled analysis of two Radiation Therapy Oncology Group trials, delays during
CRT for anal cancer were associated with increased local failure and a trend $(\mathrm{P}=0.06)$ toward increased colostomy failure (34). Thus, long waits for treatment may permit the evolution of the tumor to more advanced stages of A-SCC that would result in higher treatment failure rates (41).

Unfortunately, Non-Hispanic Black and Hispanic patients on average spent nearly two more weeks waiting for CRT treatment to start than Non-Hispanic Whites, a delay which could prompt increased psychological distress. For instance, one study found patients with newly diagnosed cancer are mentally distressed while waiting for treatment, resulting in a diminished quality of life (42). Nonetheless, most patients are willing to wait for RT if they believe that waiting will not reduce the effectiveness of treatment (43).

There are some limitations to this retrospective study. These include restriction of data to hospitals that report to the NCDB. In addition, the retrospective nature of this study does not allow us to control unobservable or unreported factors that may contribute to the TTI. Furthermore, it is impossible to determine whether delays are due to "patient factors" (e.g., transportation issues, support system, denial, etc.) or "system factors" (e.g., inaccessibility of certain services, provider cancellations, lack of streamlined process). Future projects designed to assess and improve delays would be helpful in providing further information on which factors carry the largest influence 
and which could be effectively targeted for intervention. However, strengths include the size of the NCDB database (covering approximately $85 \%$ of cancer patients in the United States), the regional diversity of providers, and the availability of matched census data allowing the incorporation of socioeconomic factors into the MVA.

In conclusion, this analysis of a large national cohort demonstrates that Non-Hispanic Black patients and Hispanic patients have a significantly longer time to treatment with CRT after being diagnosed with A-SCC compared to Non-Hispanic White patients. Although no relationship was found between TTI and OS, we could not assess the relationship between TTI and diseasefree survival, local control, or distant metastases. These endpoints merit further study to fully assess the impact of TTI on outcomes for patients with A-SCC. Further studies are also needed to determine the cause of these racial and ethnic disparities. Quality metrics or patient navigators may be helpful to track TTI and to reduce these disparities.

\section{Acknowledgements}

This work was supported by funds provided by the University of Miami Miller School of Medicine/Sylvester Comprehensive Cancer Center Department of Radiation Oncology.

\section{Footnote}

Conflicts of Interest: Dr. Portelance has disclosed that she has been a consultant for GLK, received research funding from BTG Thera Spheres, received accommodations from BTG Thera Spheres (for the Expert Users Meeting), and has a family member with stock in ViewRay. Dr. Ramey has disclosed that he has received travel accommodations from Intellisphere to attend a conference. The other authors have no conflicts of interest to declare.

Ethical Statement: The University of Miami Institutional Review Board (IRB) deemed this study exempt from review since only de-identified data was used. The American College of Surgeons granted access to the 2014 NCDB participant user file.

\section{References}

1. Howlader N, Noone AM, Krapcho M, et al. editors. SEER Cancer Statistics Review, 1975-2011. National
Cancer Institute. Based on November 2013 SEER data submission, posted to the SEER web site, April 2014. Available online: https://seer.cancer.gov/csr/1975_2011/

2. Shiels MS, Kreimer AR, Coghill AE, et al. Anal Cancer Incidence in the United States, 1977-2011: Distinct Patterns by Histology and Behavior. Cancer Epidemiol Biomarkers Prev 2015;24:1548-56.

3. NCCN clinical practice guidelines in oncology. Anal carcinoma. Version 2.2018. Available online: https://www. nccn.org/professionals/physician_gls/PDF/anal.pdf

4. Mitra D, Hong TS, Horick N, et al. Long-term outcomes and toxicities of a large cohort of anal cancer patients treated with dose-painted IMRT per RTOG 0529. Adv Radiat Oncol 2017;2:110-7.

5. Jemal A, Ward EM, Johnson CJ, et al. Annual Report to the Nation on the Status of Cancer, 1975-2014, Featuring Survival. J Natl Cancer Inst 2017;109. doi: 10.1093/jnci/ djx030.

6. Baughman DM, Shah BK. Disparities in receipt of radiotherapy and survival by age, sex, and race among patients with non-metastatic squamous cell carcinoma of the anus. J Gastrointest Oncol 2016;7:968-73.

7. Murphy CT, Galloway TJ, Handorf EA, et al. Survival Impact of Increasing Time to Treatment Initiation for Patients With Head and Neck Cancer in the United States. J Clin Oncol 2016;34:169-78.

8. Bleicher RJ, Ruth K, Sigurdson ER, et al. Time to Surgery and Breast Cancer Survival in the United States. JAMA Oncol 2016;2:330-9.

9. Daling JR, Madeleine MM, Johnson LG, et al. Human papillomavirus, smoking, and sexual practices in the etiology of anal cancer. Cancer 2004;101:270-80.

10. Ang KK, Harris J, Wheeler R, et al. Human papillomavirus and survival of patients with oropharyngeal cancer. $\mathrm{N} \mathrm{Engl}$ J Med 2010;363:24-35.

11. Termine N, Panzarella V, Falaschini S, et al. HPV in oral squamous cell carcinoma vs head and neck squamous cell carcinoma biopsies: a meta-analysis (1988-2007). Ann Oncol 2008;19:1681-90.

12. Rettig EM, D'Souza G. Epidemiology of head and neck cancer. Surg Oncol Clin N Am 2015;24:379-96.

13. Trott KR, Kummermehr J. What is known about tumour proliferation rates to choose between accelerated fractionation or hyperfractionation? Radiother Oncol 1985;3:1-9.

14. Gorin SS, Heck JE, Cheng B, et al. Delays in breast cancer diagnosis and treatment by racial/ethnic group. Arch Intern Med 2006;166:2244-52. 
15. Stokes WA, Hendrix LH, Royce TJ, et al. Racial differences in time from prostate cancer diagnosis to treatment initiation: a population-based study. Cancer 2013;119:2486-93.

16. Murphy CT, Galloway TJ, Handorf EA, et al. Increasing time to treatment initiation for head and neck cancer: an analysis of the National Cancer Database. Cancer 2015;121:1204-13.

17. Caudell JJ, Locher JL, Bonner JA. Diagnosis-to-treatment interval and control of locoregionally advanced head and neck cancer. Arch Otolaryngol Head Neck Surg 2011;137:282-5.

18. Kulkarni GS, Urbach DR, Austin PC, et al. Longer wait times increase overall mortality in patients with bladder cancer. J Urol 2009;182:1318-24.

19. Naghavi AO, Echevarria MI, Grass GD, et al. Having Medicaid insurance negatively impacts outcomes in patients with head and neck malignancies. Cancer 2016;122:3529-37.

20. American College of Surgeons Commission on Cancer. American College of Surgeons: National Cancer Database: Tools, Reports, and Resources. 2016. Available online: https://www.facs.org/ /media/files/quality\%20programs/ cancer/ncdb/ncdb_2016_booklet_v11.ashx

21. Bilimoria KY, Stewart AK, Winchester DP, et al. The National Cancer Data Base: a powerful initiative to improve cancer care in the United States. Ann Surg Oncol 2008;15:683-90.

22. Boffa DJ, Rosen JE, Mallin K, et al. Using the National Cancer Database for Outcomes Research: A Review. JAMA Oncol 2017;3:1722-8.

23. Winchester DP, Stewart AK, Phillips JL, et al. The national cancer data base: past, present, and future. Ann Surg Oncol 2010;17:4-7.

24. World Health Organization. International classification of diseases for oncology: ICD-O-3. Available online: http:// codes.iarc.fr/

25. American College of Surgeons. Getting Started-A User's Guide. Available online: http://ncdbpuf.facs.org/node/274

26. Go RS, Bartley AC, Crowson CS, et al. Association Between Treatment Facility Volume and Mortality of Patients With Multiple Myeloma. J Clin Oncol 2017;35:598-604.

27. Deyo RA, Cherkin DC, Ciol MA. Adapting a clinical comorbidity index for use with ICD-9-CM administrative databases. J Clin Epidemiol 1992;45:613-9.

28. van Harten MC, Hoebers FJ, Kross KW, et al. Determinants of treatment waiting times for head and neck cancer in the Netherlands and their relation to survival. Oral Oncol 2015;51:272-8.

29. Bilimoria KY, Ko CY, Tomlinson JS, et al. Wait times for cancer surgery in the United States: trends and predictors of delays. Ann Surg 2011;253:779-85.

30. Devoe JE, Baez A, Angier H, et al. Insurance + access not equal to health care: typology of barriers to health care access for low-income families. Ann Fam Med 2007;5:511-8.

31. Strohl AE, Feinglass JM, Shahabi S, et al. Surgical wait time: A new health indicator in women with endometrial cancer. Gynecol Oncol 2016;141:511-5.

32. Smedley BD, Stith AY, Nelson AR. Unequal Treatment: Confronting Racial and Ethnic Disparities in Health Care. Washington, DC: The National Academies Press, 2003.

33. Cook MB, McGlynn KA, Devesa SS, et al. Sex disparities in cancer mortality and survival. Cancer Epidemiol Biomarkers Prev 2011;20:1629-37.

34. Ben-Josef E, Moughan J, Ajani JA, et al. Impact of overall treatment time on survival and local control in patients with anal cancer: a pooled data analysis of Radiation Therapy Oncology Group trials 87-04 and 98-11. J Clin Oncol 2010;28:5061-6.

35. McLaughlin JM, Anderson RT, Ferketich AK, et al. Effect on survival of longer intervals between confirmed diagnosis and treatment initiation among low-income women with breast cancer. J Clin Oncol 2012;30:4493-500.

36. Nanthamongkolkul K, Hanprasertpong J. Longer waiting times for early stage cervical cancer patients undergoing radical hysterectomy are associated with diminished longterm overall survival. J Gynecol Oncol 2015;26:262-9.

37. E C, Dahrouge S, Samant R, et al. Radical radiotherapy for cervix cancer: the effect of waiting time on outcome. Int J Radiat Oncol Biol Phys 2005;61:1071-7.

38. Chen PC, Liu WS, Huang WL, et al. The impact of time factors on overall survival in patients with nasopharyngeal carcinoma: a population-based study. Radiat Oncol 2016;11:62.

39. Mackillop WJ, Bates JH, O'Sullivan B, et al. The effect of delay in treatment on local control by radiotherapy. Int J Radiat Oncol Biol Phys 1996;34:243-50.

40. Kishan AU, Cui J, Wang PC, et al. Quantification of gross tumour volume changes between simulation and first day of radiotherapy for patients with locally advanced malignancies of the lung and head/neck. J Med Imaging Radiat Oncol 2014;58:618-24.

41. Roohipour R, Patil S, Goodman KA, et al. Squamouscell carcinoma of the anal canal: predictors of treatment 
outcome. Dis Colon Rectum 2008;51:147-53.

42. Visser MR, van Lanschot JJ, van der Velden J, et al. Quality of life in newly diagnosed cancer patients waiting for surgery is seriously impaired. J Surg Oncol
2006;93:571-7.

43. Lehman M, Jacob S, Delaney G, et al. Waiting times for radiotherapy--a survey of patients' attitudes. Radiother Oncol 2004;70:283-9.

Cite this article as: Ramey SJ, Rich BJ, Kwon D, Mellon EA, Wolfson A, Portelance L, Yechieli R. Demographic disparities in delay of definitive chemoradiation for anal squamous cell carcinoma: a nationwide analysis. J Gastrointest Oncol 2018;9(6):1109-1126. doi: 10.21037/jgo.2018.08.07 


\section{Supplementary}

A

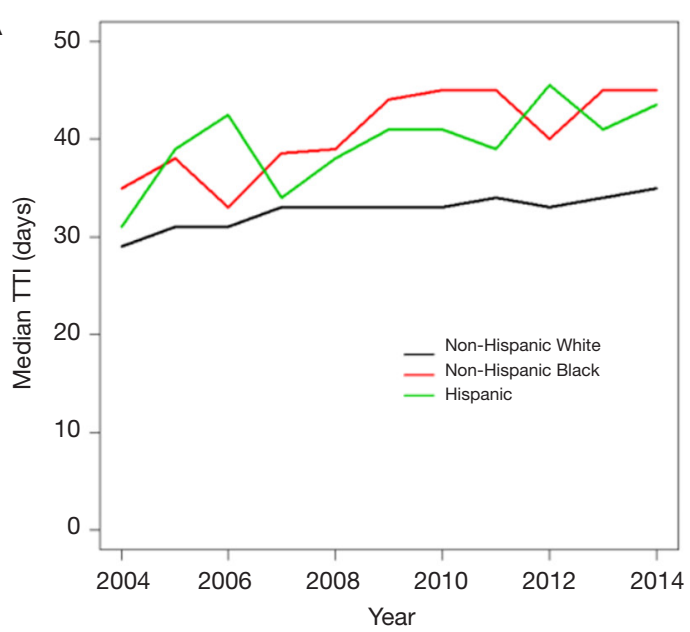

C

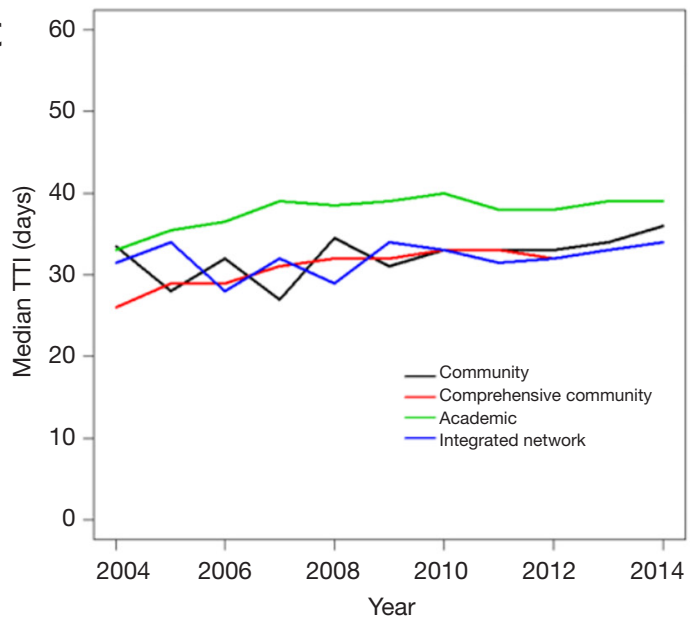

B
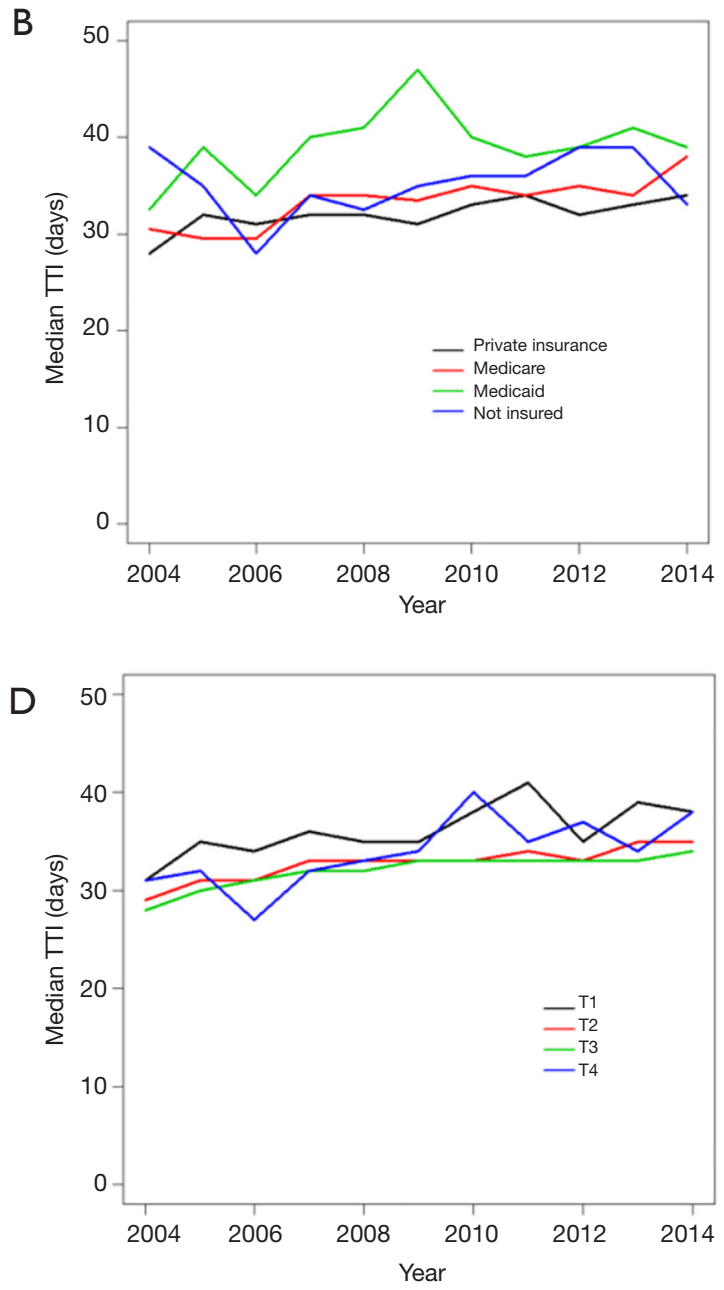

Figure S1 Changes in median time to treatment initiation over time (years) stratified by race and ethnicity (A), insurance status (B), reporting facility type (C), and AJCC clinical tumor classification (D). TTI, time to treatment initiation; AJCC, American Joint Committee on Cancer. 
$7,233(100.0)+3$

Hispanic

Non-Hispanic Black

AAPI

Other/unknown

Gender

Male

Female

Year of diagnosis

2004

2005

2000

2007

2008

2009

2010

2011

2012

2013

Age (yea
$>70$

$>70$

$51-60$

41-50

$\leq 40$

Insurance type

Private insurrace

Medicaid

Not insured

Other/unknown

$\begin{array}{lll}5,803(80.2) & 36.6 & \\ 332(4.6) & 47.6 & 39 \\ 629(8.7) & 49.2 & 40\end{array}$

$629(8.7) \quad 49.2$

$75(1.0) \quad 42.6-35$

$394(5.4) \quad 35.5 \quad 31$

$2,168(30.0) \quad 41.5 \quad 35$

$5,065(70.0) \quad 36.8$

$\begin{array}{lll}377(5.2) & 36.2 & 31\end{array}$

$\begin{array}{lll}409(5.7) & 37.1 & 33\end{array}$

$\begin{array}{lll}470(6.5) & 35.8 & 31 \\ 594(8.2) & 37.7 & 33\end{array}$

$\begin{array}{lll}595(8.2) & 37.7 & 33\end{array}$

$781(108) \quad 372-33$

$\begin{array}{lll}808(11.2) & 39.5 & 34\end{array}$

36 (12.9) $38.5 \quad 34$

$1046(145)(25)$

$1,173(16.2) \quad 37.3$

$\begin{array}{lll}1,742(24.1) & 37.8 & 34\end{array}$

$\begin{array}{lll}2,599(35.9) & 38.4 & 34\end{array}$

$\begin{array}{lll}1,444(20.0) & 38.4 & 33\end{array}$

$\begin{array}{lll}275(3.8) & 41.9 & 33\end{array}$

$\begin{array}{lll}3,576(49.4) & 36.1 & 32\end{array}$

$\begin{array}{lll}2,327(32.2) & 37.9 & 34\end{array}$

$\begin{array}{lll}596(8.2) & 46.3 & 39\end{array}$

$\begin{array}{lll}496(6.9) & 42.8 & 36\end{array}$

$\begin{array}{lll}238(3.3) & 43.3 & 37\end{array}$

$\geq 63,000$

48,000-62,999

$38,000-47,999$

$\begin{array}{lll}2,103(29.1) & 36.7 & 32 \\ 1.954(27.0) & 37.9 & 33\end{array}$

$\begin{array}{lll}1,803(24.9) & 38.7 & 33\end{array}$

$\begin{array}{lll}1,321(18.3) & 40.4 & 34\end{array}$

$<38,000$

$52(0.7) \quad 37.1$

Education status (\% without high school diploma) in zip code of patient residence

$\begin{array}{llll}<7.0 & 1,765(24.4) & 35.9 & 32 \\ 7.0-12.9 & 2,363(32.7) & 37.3 & 33\end{array}$

$13-20.9$

$\geq 21.0$

Unknown

Metropolitan $\geq 1$ million

Metropolitan $<1$ million

Urban

Rural

Unknown

Distance to reporting center (miles)

$\leq 10$
$11-20$

$11-20$
$21-50$

$>50$

Reporting facility type

$\begin{array}{lll}1,931(26.7) & 39.2 & 34\end{array}$

$124(15.5) \quad 42.2 \quad 36$

$<0.001$

0.658

$\begin{array}{lll}3.685(50.9) & 39.8 & 34\end{array}$

$\begin{array}{lll}39.8 & 31(31.0) \quad 36.2 & 32\end{array}$

$1,035(14.3) \quad 36.7 \quad 34$

$\begin{array}{lll}113(1.6) & 39.3 & 32\end{array}$

$159(2.2)$

$\quad 38.0 \quad 33$

$\begin{array}{lll}1,490(20.6) & 37.4 & 33\end{array}$

$\begin{array}{lll}1,146(15.8) & 38.4 & 33.5 \\ 500(6.9) & 41.7 & 39\end{array}$

$<0.001$

$<0.001$

$\begin{array}{lll}702(9.7) & 36.4 & 32\end{array}$

$3,207(44.3) \quad 35.0 \quad 32$

$\begin{array}{lll}2.300(31.8) & 43.2 & 38\end{array}$

$802(11.1) \quad 36.6$

$222(3.1) \quad 43.3$

$497(6.9) \quad 35.6 \quad 32$

$\begin{array}{lll} & 36.5 \\ 5,246(72.5) & 38.9\end{array}$

Lower tertile

Upper tertio

Reporting facility location

Northeast

West

Unknown

No

Unknown

Non-IMRT, non-proton

IMRT, proton

Charson/Deyo comorbidity score

(1)

1

Clinical tumor classification

$\mathrm{T} 1$

T2

To/15

Unknown

Clinical nodal classification

$1,446(20.0) \quad 41.8 \quad 38$

$\begin{array}{lll}2,425(33.5) & 38.0 & 33 \\ 1.947(26.9) & 34.1 & 31\end{array}$

$\begin{array}{lll}1,193(16.5) & 40.0 & 31 \\ 222 & 4.93\end{array}$

$222(3.1) \quad 43.3 \quad 34$

$\begin{array}{lll}1.278(17.7) & 35.9 & 32\end{array}$

$<0.00$

$<0.001$

, $045(14.4) \quad 41.4$

3794

$\begin{array}{lll}1.684(23.3) & 36.4 & 32\end{array}$

$\begin{array}{lll}599(8.3) & 40.4 & 34\end{array}$

$\begin{array}{lll}5(0.1) & 38.4 & 28 \\ & 45.1 & 39\end{array}$

(15)

4,569 (63.2) $\quad 37.9$

$\quad 37.0 \quad 33$

$\begin{array}{lll}38.7 & 34\end{array}$

$$
\text { Unknown }
$$$$
35.4
$$ 